\title{
MRgHIFU treatment in pain palliation of bone metastases: initial experience from the Rizzoli Orthopaedic Institute
}

\author{
Alberto Bazzocchi ${ }^{1,2^{*}}$, Alessandro Napoli ${ }^{3}$, Paolo Spinnato ${ }^{1}$, Giancarlo Facchini ${ }^{2}$, Danila Diano ${ }^{2}$, Maurizio Busacca ${ }^{1}$, \\ Carlo Catalano ${ }^{3}$, Ugo Albisinni ${ }^{1}$
}

From 2nd European Symposium on Focused Ultrasound Therapy

Rome, Italy. 10-11 October 2013

\section{Background}

The increasing longevity of the population coupled with the progress of treatments for primary tumors is enhancing the incidence and the burden of distant metastases such as bone metastases. Bone metastases are the most common cause of cancer-related pain. Quality of life may be significantly impaired as a consequence of painful bone metastases. The treatment of secondary bone lesions will become even more important in the near future. The development of strategies to improve quality of life in patients with bone metastases is fundamental and represents a major clinical challenge. This issue is of huge impact on today health care and economy.

\section{Purpose of the study}

The aim of this work was to assess the efficacy of MRgHIFU in the treatment of pain caused by bone metastases, in the preliminary cohort of patient treated at our Institute.

\section{Materials and methods}

From November 2012, 11 patients affected by bone metastases were enrolled in the study. Pain was scored before and after MRgHIFU (ExAblate 2100, InSightec, Israel on 1.5 T Signa Twin Speed MR system, GE, USA) using visual analogue scale (VAS, $0-10$ points). The initial follow-up included clinical assessment at 1 and 3 months.

\section{Results}

Fourteen lesions were treated in 11 patients ( 6 males, 5 females - age 51 \pm , range 47-68 years old) with different primary cancers ( 4 kidney, 3 breast, 2 lung, 1 thyroid, 1 colon). The treated lesions were located as follows: 11 at pelvis, 2 at femur, 1 at rib. In four patients the bone lesion was single. Treatments were performed under spinal anesthesia in 9/11 patients, under general anesthesia in $2 / 11$. One patient died two months after the treatment for causes not related to the procedure, while five patients reached the 3-month check point. After 1 month, the average pain drop amounted to $56 \%(\mathrm{p}<0.01)$, and after 3 months to $69 \%(\mathrm{p}<0.01)$ with 3 out of 5 patients presenting VAS $=0$. The only experienced complication was prostate inflammation with urinary retention after the treatment of a pubic lesion.

\section{Conclusion}

Our preliminary experience confirms the efficacy of MRgFUS in controlling pain of bone metastases.

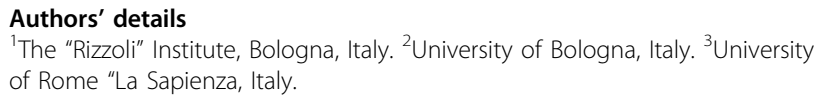

Published: 10 December 2014

doi:10.1186/2050-5736-2-S1-A15

Cite this article as: Bazzocchi et al:: MRgHIFU treatment in pain palliation of bone metastases: initial experience from the Rizzoli Orthopaedic Institute. Journal of Therapeutic Ultrasound 2014 2(Suppl 1): A15.

"The "Rizzoli" Institute, Bologna, Italy

Full list of author information is available at the end of the article 